\title{
The future of automotive radar - Leveraging metamaterial antennas and intelligent algorithms
}

\author{
Armin Volkel, C. Marx \\ Metawave Corporation
}

This manuscript is not available according to publishing restriction. Thank you for your understanding. 